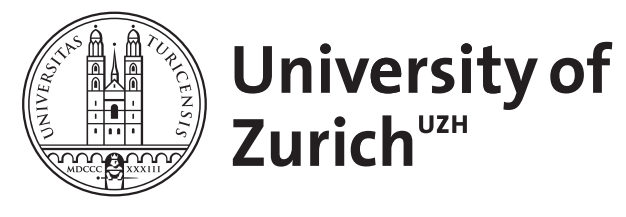

\title{
Canonical rational equivalence of intersections of divisors
}

Kresch, A

\begin{abstract}
We consider the operation of intersecting with a locally principal Cartier divisor (i.e., a Cartier divisor which is principal on some neighborhood of its support). We describe this operation explicitly on the level of cycles and rational equivalences and as a corollary obtain a formula for rational equivalence between intersections of two locally principal Cartier divisors. Such canonical rational equivalence applies quite naturally to the setting of algebraic stacks. We present two applications: (i) a simplification of the development of Fulton-MacPherson-style intersection theory on Deligne-Mumford stacks, and (ii) invariance of a key rational equivalence under a certain group action (which is used in developing the theory of virtual fundamental classes via intrinsic normal cones).
\end{abstract}

DOI: https://doi.org/10.1007/s002220050317

Posted at the Zurich Open Repository and Archive, University of Zurich

ZORA URL: https://doi.org/10.5167/uzh-22142

Journal Article

Accepted Version

Originally published at:

Kresch, A (1999). Canonical rational equivalence of intersections of divisors. Inventiones Mathematicae, 136(3):483-496.

DOI: https://doi.org/10.1007/s002220050317 


\title{
Canonical rational equivalence of intersections of divisors
}

\author{
Andrew Kresch ${ }^{1}$
}

3 December 1997

\section{Introduction}

One way to define an operation in intersection theory is to define a map on the group of algebraic cycles together with a map on the group of rational equivalences which commutes with the boundary operation. Assuming the maps commute with smooth pullback, the extension of the operation to the setting of algebraic stacks is automatic. The goal of the first section of this paper is to present the operation of intersecting with a principal Cartier divisor in this light.

We then show how this operation lets us obtain a rational equivalence which is fundamental to intersection theory. A one-dimensional family of cycles on an algebraic variety always admits a unique limiting cycle, but a family of cycles over the punctured affine plane may yield different limiting cycles if one approaches the origin from different directions. An important step in the historical development of intersection theory was realizing how to prove that any two such limiting cycles are rationally equivalent. The results of the first section yield, as a corollary, a new, explicit formula for this rational equivalence.

\footnotetext{
${ }^{1}$ Funded by a Fannie and John Hertz Foundation Fellowship for Graduate Study and an Alfred P. Sloan Foundation Dissertation Fellowship.
} 
Another important rational equivalence in intersection theory is the one that is used to demonstrate commutativity of Gysin maps associated to regularly embedded subschemes. In section 2, we exhibit a two-dimensional family of cycles such that the cycles we obtain from specializing in two different ways are precisely the ones we need to show to be rationally equivalent to obtain the commutativity result. Our explicit rational equivalence respects smooth pullback, and hence the generalization to stacks is automatic. This simplifies intersection theory on Deligne-Mumford stacks as in [7], where construction of such a rational equivalence fills the most difficult section of that important paper.

Since our rational equivalence arises by considering families of cycles on a larger total space, we are able to deduce (section 3 ) that the rational equivalence is invariant under a certain naturally arising group action. The key observation is that we can manipulate the situation so that the group action extends to the total space. This equivariance result is used, but appears with mistaken proof, in [2], where an important new tool of modern intersection theory — the theory of virtual fundamental classes — is developed.

The author would like to thank S. Bloch, W. Fulton, T. Graber, and R. Pandharipande for helpful advice and the organizers and staff of the MittagLeffler Institute for hospitality during the 1996-97 program in algebraic geometry.

\section{Intersection with divisors}

In this section we work exclusively on schemes of finite type over a fixed base field. The term variety denotes integral scheme, and by a subvariety we mean an integral closed subscheme. We denote by $Z_{*} X, W_{*} X$, and $A_{*} X$, respectively, the group of algebraic cycles, group of rational equivalences, and Chow group of a scheme $X$. The boundary map $W_{*} X \rightarrow Z_{*} X$ is denoted $\partial$. We refer to [3] for basic definitions and properties from intersection theory. Given a Cartier divisor $D$ we denote by $[D]$ the associated Weil divisor (it is important to note that the notion of Weil divisor makes sense on arbi-

trary varieties, [3] $\S 1.2$ ). If $X$ is a variety then we denote by $X^{1}$ the set of subvarieties of codimension 1 . 
Definition 1.1. Let $X$ be a variety and let $D$ be a Cartier divisor. Let $\pi: \widehat{X} \rightarrow X$ be the normalization map. The support of $D$, denoted $|D|$, is defined to be $\pi\left(\bigcup_{\substack{\operatorname{ord} W \\ W} \pi^{1} D \neq 0} W\right)$.

Remark 1.2. This agrees with the naïve notion of support (the union of all subvarieties appearing with nonzero coefficient in $[D]$ ) when $X$ is normal or when $D$ is effective.

Remark 1.3. There is yet another notion of support which appears in [3]. There, the support of a divisor is a piece of data that must be specified along with the divisor. Given a Cartier divisor $D$ on a variety $X$, let $Z$ be any closed subscheme such that away from $Z$ the canonical section of $\mathcal{O}(D)$ is well-defined and nonvanishing. Then, [3] defines an intersection operation $A_{k}(X) \rightarrow A_{k-1}(Z)$. Unfortunately, the support $|D|$ which we have defined is not generally a support in this sense. Hence in the definition below we require that our divisors be specified by defining functions which are regular away from their supports.

We shall denote by $|D|^{0}$ the set of irreducible components of $|D|$.

Definition 1.4. Let $X$ be a variety. A $P$-divisor on $X$ is a tuple $\left(U, U^{\prime}, x\right)$ such that

(i) $U$ and $U^{\prime}$ are nonempty open subschemes of $X$ such that $U \cup U^{\prime}=X$;

(ii) $x \in k(U)^{*}$;

(iii) $\left.x\right|_{U \cap U^{\prime}} \in \mathcal{O}^{*}\left(U \cap U^{\prime}\right)$; and

(iv) the data $\left(x \in k(U)^{*}, 1 \in k\left(U^{\prime}\right)^{*}\right)$ specifies a Cartier divisor $D$ such that $|D|=X \backslash U^{\prime}$.

By abuse of terminology, we call $D$ a $P$-divisor if $D$ is the Cartier divisor associated to a $P$-divisor as in (iv). Given a $P$-divisor as above, we call $x$ the local defining function. A $P$-divisor may be pulled back via a morphism of varieties provided that the image of the morphism is not contained in the support of the underlying Cartier divisor.

Examples. (i) Let $X$ be a normal variety. Let $x \in k(X)^{*}$ specify a principal Cartier divisor $D$. Then $(X, X \backslash|D|, x)$ is a $P$-divisor. 
(ii) Let $X$ be a variety. Every effective principal Cartier divisor is a $P$ divisor.

(iii) Let $X$ be a variety, and let $\pi: X \rightarrow \mathbb{P}^{1}$ be a dominant morphism. Then the fiber of $\pi$ over $\{0\}$ is a $P$-divisor.

The operation of intersecting with a Cartier divisor is generally defined only on the level of rational equivalence classes of cycles. When $V \subset|D|$, we have $D \cdot[V]=c_{1}\left(\left.\mathcal{O}(D)\right|_{V}\right) \cap[V]$, and there is generally no way to pick canonically a cycle representing this first Chern class. The exception is when $\left.\mathcal{O}(D)\right|_{|D|}$ is trivial, or in our terminology, $D$ is a $P$-divisor. Then, we may define a cycle-level intersection operation (see [3], Remark 2.3).

Definition 1.5. Let $X$ be a variety, and let $D$ be a $P$-divisor on $X$. The cycle-level intersection operation

$$
D \cdot-: Z_{k}(X) \rightarrow Z_{k-1}(|D|)
$$

is given by

$$
D \cdot[V]= \begin{cases}{\left[\left.D\right|_{V}\right]} & \text { if } V \not \subset|D| \\ 0 & \text { if } V \subset|D|\end{cases}
$$

The claim that this map passes to rational equivalence and hence gives an intersection operation $D \cdot: A_{k}(X) \rightarrow A_{k-1}(|D|)$ is proved in [3], but not in a way that makes it easy to see how $D \cdot \alpha$ is to be rationally equivalent to zero if $\alpha$ is a cycle that is rationally equivalent to zero. Following the program set out in the introduction, we would like to demonstrate this fact by giving an explicit map on rational equivalences which commutes with the boundary operation.

Definition 1.6. Let $X$ be a variety, and let $D$ be a $P$-divisor on $X$ with local defining function $x$. Say $V$ is a subvariety of $X$ with normalization $\pi: \widehat{V} \rightarrow V$, and suppose $y \in k(V)^{*}$. We define the intersection operation on the level of rational equivalences

$$
D \cdot-: W_{k}(X) \rightarrow W_{k-1}(|D|)
$$

by

$$
D \cdot y= \begin{cases}\pi_{*}\left(\left.\bigoplus_{W \in\left|\pi^{*} D\right|^{0}}\left(y^{\operatorname{ord}_{W} x} / x^{\mathrm{ord}_{W} y}\right)\right|_{W}\right) & \text { if } V \not \subset|D| \\ 0 & \text { if } V \subset|D|\end{cases}
$$


Here, $\pi_{*}: W_{*} \widehat{V} \rightarrow W_{*} V$ is pushforward of rational equivalence.

Remark 1.7. This definition explains why we a require the definition of a $P$ divisor to include more data than just that of the underlying Cartier divisor. The map (1) actually depends on the choice of defining function.

Proposition 1.8. Let $X$ be a variety and let $D$ be a $P$-divisor on $X$. Then the diagram

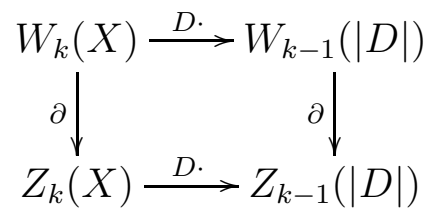

commutes.

This follows easily from

Proposition 1.9. Let $X$ be a normal variety and let $x$ and $y$ be rational functions with associated principal Cartier divisors $D$ and $E$. For $V \in X^{1}$ set $a_{V}=\operatorname{ord}_{V} x$ and $b_{V}=\operatorname{ord}_{V} y$. Then

$$
\begin{aligned}
\sum_{V \in X^{1}} \partial\left(y^{a_{V}} /\left.x^{b_{V}}\right|_{V}\right) & =0 \\
\partial(D \cdot y) & =D \cdot(\partial y) ; \\
D \cdot[E]-E \cdot[D] & =\sum_{V \in|D|^{0} \cap|E|^{0}} \partial\left(y^{a_{V}} /\left.x^{b_{V}}\right|_{V}\right) .
\end{aligned}
$$

Proof. If we split the sum in (2) into a sum over $V \in|D|^{0}$ and a sum over $V \notin|D|^{0}$ we obtain (3). Similarly if we split away the terms with $V \in|D|^{0} \cap|E|^{0}$ we obtain (4) from (2). So, for a fixed variety $X$ and fixed divisors $D$ and $E$, the three assertions are equivalent. Now, we get (2) as a consequence of the tame symbol in $K$-theory, cf. [6] $\S 7$, or by the following elementary geometric argument. We quickly reduce to the case where $D$ and $E$ are effective. Then, when $D$ and $E$ meet properly, (4) follows from [3], Theorem 2.4, case 1. An induction on excess of intersection

$$
\varepsilon(D, E)=\max _{V \in X^{1}} a_{V} \cdot b_{V}
$$


completes the proof: if we denote the normalized blow-up along the ideal $(x, y)$ by $\sigma: X^{\prime} \rightarrow X$ and denote the exceptional divisor by $Z$ then we may write $\sigma^{*} D=Z+D^{\prime}$ and $\sigma^{*} E=Z+E^{\prime}$, and now $\left|D^{\prime}\right| \cap\left|E^{\prime}\right|=\emptyset$ and $\max \left(\varepsilon\left(D^{\prime}, Z\right), \varepsilon\left(E^{\prime}, Z\right)\right)<\varepsilon(D, E)$ (assuming $D$ and $E$ do not meet properly), cf. [3], Lemma 2.4. The result pushes forward.

Corollary 1.10. Let $D$ and $E$ be $P$-divisors on a variety $X$, with respective local defining functions $x$ and $y$. Let $\pi: \widehat{X} \rightarrow X$ be the normalization map. Then

$$
D \cdot[E]-E \cdot[D]=\partial \omega
$$

where $\omega \in W_{*}(|D| \cap|E|)$ is given by

$$
\omega=\sum_{V \in\left|\pi^{*} D\right|^{0} \cap\left|\pi^{*} E\right|^{0}} \pi_{*}\left(y^{\operatorname{ord}_{V} x} /\left.x^{\operatorname{ord}_{V} y}\right|_{V}\right) .
$$

\section{Application to intersection theory on stacks}

All stacks (and schemes) in this section are algebraic stacks of Artin type, [1], [5], which are locally of finite type over the base field. The notion of $P$-divsor on a stack makes sense (it is as in Definition 1.4 with "open subscheme" replaced by "open substack," where by "Cartier divisor" in part (iv) of the definition we mean a global section of the sheaf $\mathcal{K}^{*} / \mathcal{O}^{*}$ for the Zariski topology, and where normalization, order along a substack of codimension 1 , and support of a Cartier divisor are well defined on stacks because they all respect smooth pullback and hence can be defined locally). Since an Artin stack possesses a smooth cover by a scheme, the operation of intersecting with a $P$-divisor on a stack comes for free once we know that this operation on schemes commutes with smooth pullback. Also for free we get Corollary 1.10 in the setting of stacks: the formation of $\omega$ from $X, D$, and $E$ commutes with smooth pullback.

Proposition 2.1. Let $X$ be a variety, let $Y$ be a scheme, and let $f: Y \rightarrow X$ be a smooth morphism. Let $D$ be a $P$-divisor on $X$. Then $f^{*} \circ D \cdot=\left(f^{*} D\right) \cdot \circ f^{*}$, both as maps on cycles and as maps on rational equivalences. 
We now turn to an application of Corollary 1.10 to intersection theory on Deligne-Mumford stacks (where a reasonable intersection theory exists, cf. [4], [7]). Central to intersection theory on schemes is the Gysin map corresponding to a regularly embedded subscheme, since the diagonal of a smooth scheme is a regular embedding and this way we obtain an intersection product on smooth schemes. The diagonal morphism for a smooth DeligneMumford stack is not generally an embedding, but it is representable and unramified.

Lemma 2.2. Let $f: F \rightarrow G$ be a representable morphism of Artin stacks. Then $f$ is unramified if and only if there exists a commutative diagram

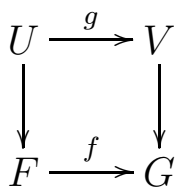

such that the vertical maps are smooth surjective, $g$ is a closed immersion of schemes, and the induced morphism $U \rightarrow F \times{ }_{G} V$ is étale.

Proof. This is [7], Lemma 1.19. Because this is such a basic fact about properties of morphisms in algebraic geometry, we present an elementary proof in the Appendix.

To describe a representable morphism, we use the terminology local immersion as a synonym for unramified and call $f$ above a regular local immersion if moreover $g$ is a regular embedding of schemes. Since formation of normal cone is of a local nature, an obvious patching construction produces the normal cone $C_{X} Y$ to a local immersion $X \rightarrow Y$; the cone is a bundle in case $X \rightarrow Y$ is a regular local immersion.

To get Fulton-MacPherson-style intersection theory on Deligne-Mumford stacks we clearly need to have Gysin maps for regular local immersions. In [7], the author supplies this needed Gysin map by giving a (long, difficult) proof of the stack analogue of [3], Theorem 6.4, namely

Proposition 2.3. Let $X \rightarrow Y$ and $Y^{\prime} \rightarrow Y$ be local immersions of Artin stacks. Then $\left[C_{X \times{ }_{Y} C_{Y^{\prime}} Y} C_{Y^{\prime}} Y\right]=\left[C_{C_{X} Y \times_{Y} Y^{\prime}} C_{X} Y\right]$ in $A_{*}\left(C_{X} Y \times{ }_{Y} C_{Y^{\prime}} Y\right)$. 
Remark 2.4. Though our focus is on applications to intersection theory on Deligne-Mumford stacks, we continue to make use of constructions which behave well locally with respect to smooth pullback, and hence our results are valid in the more general setting of Artin stacks.

Remark 2.5. Given a stack $X$ which is only locally of finite type over a base field, we must take $Z_{*} X$ to be the group of locally finite formal linear combinations of integral closed substacks. More intrinsically, $Z_{*} X$ is the group of global sections of the sheaf for the smooth topology $\mathcal{Z}_{*}$ which associates to a stack of finite type the free abelian group on integral closed substacks. Similarly, $W_{*} X$ is the group of global sections of sheaf $\mathcal{W}_{*}$. As always, $A_{*} X$ is defined to be $Z_{*} X / \partial W_{*} X$.

The methods of the last section allow us to supply a new, simpler proof of this proposition.

Proof. Recall that given a closed immersion $X \rightarrow Y$ there are associated spaces

$$
\begin{aligned}
& M_{X} Y=\mathrm{B} \ell_{X \times\{0\}} Y \times \mathbb{P}^{1}, \\
& M_{X}^{\circ} Y=M_{X} Y \backslash \mathrm{B} \ell_{X \times\{0\}} Y \times\{0\},
\end{aligned}
$$

cf. [3] §5.1. Given a locally closed immersion, say with $U$ is an open subscheme of $Y$ and $X$ a closed subscheme of $U$, then $M_{X}^{\circ} Y:=M_{X}^{\circ} U \amalg_{U \times \mathbb{A}^{1}} Y \times$ $\mathbb{A}^{1}$ makes sense and is independent of the choice of $U$.

This lets us define $M_{F}^{\circ} G$ when $F \rightarrow G$ is a local immersion of stacks, as follows. Assume we have a diagram as in the statement of Lemma 2.2, and set $R=U \times_{F} U$ and $S=V \times_{G} V$. There are projections $q_{1}, q_{2}: S \rightarrow G$. Define $s_{i}: M_{R}^{\circ} S \rightarrow M_{U}^{\circ} V(i=1,2)$ to be the composite $M_{R}^{\circ} S \rightarrow M_{U \times{ }_{G} V}^{\circ} S \rightarrow M_{U}^{\circ} V$, where the first map is induced by the open immersion $R \rightarrow U \times_{G} V$ and the second, by pullback via $q_{i}$. Then $\left[M_{R}^{\circ} S \rightrightarrows M_{U}^{\circ} V\right]$ is the smooth groupoid presentation of a stack which we denote $M_{F}^{\circ} G$. We have, by descent, a morphism $M_{F}^{\circ} G \rightarrow \mathbb{P}^{1}$, which is flat and has as general fiber a copy of $G$ and as special fiber the normal cone $C_{F} G$.

In the situation at hand, this construction gives

$$
(s \times t): M_{X}^{\circ} Y \times_{Y} M_{Y^{\prime}}^{\circ} Y \rightarrow \mathbb{P}^{1} \times \mathbb{P}^{1}
$$


and hence a pair of $P$-divisors, $D$ (corresponding to $s$ ) and $E$ (corresponding to $t)$. We note that $(s \times t)^{-1}(\{0\} \times\{0\})=C_{X} Y \times_{Y} C_{Y^{\prime}} Y$. Since the restriction of $s \times t$ to $\mathbb{P}^{1} \times \mathbb{P}^{1} \backslash\{0\} \times\{0\}$ is flat, we have

$$
\begin{aligned}
& {[D]=\left[C_{X} Y \times_{Y} M_{Y^{\prime}}^{\circ} Y\right] \bmod Z_{*}\left(C_{X} Y \times_{Y} C_{Y^{\prime}} Y\right),} \\
& {[E]=\left[M_{X}^{\circ} Y \times_{Y} C_{Y^{\prime}} Y\right] \bmod Z_{*}\left(C_{X} Y \times_{Y} C_{Y^{\prime}} Y\right) .}
\end{aligned}
$$

We examine the fiber of $s \times t$ over $\mathbb{P}^{1} \times\{0\}$ more closely. The fiber square

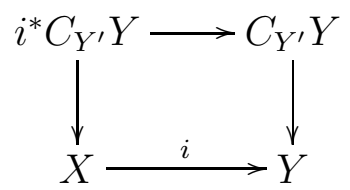

gives rise to a closed immersion $f$ making

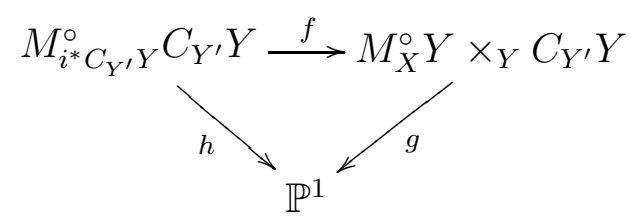

commute (where $g$ is first projection followed by $s$ ). Since $f$ is an isomorphism away from the fiber over 0 , we see in fact that

$$
[E]=\left[M_{i^{*} C_{Y^{\prime}} Y}^{\circ} C_{Y^{\prime}} Y\right] \quad \bmod Z_{*}\left(C_{X} Y \times_{Y} C_{Y^{\prime}} Y\right),
$$

and since $h$ is flat we find

$$
D \cdot[E]=\left[C_{i^{*} C_{Y^{\prime}} Y} C_{Y^{\prime}} Y\right] .
$$

Similarly, if $j$ denotes the map $Y^{\prime} \rightarrow Y$ then

$$
E \cdot[D]=\left[C_{j^{*} C_{X} Y} C_{X} Y\right]
$$

and so the rational equivalence $\omega \in W_{*}\left(C_{X} Y \times_{Y} C_{Y^{\prime}} Y\right)$ of Corollary 1.10 satisfies

$$
\partial \omega=\left[C_{X \times_{Y} C_{Y^{\prime}} Y} C_{Y^{\prime}} Y\right]-\left[C_{C_{X} Y \times_{Y} Y^{\prime}} C_{X} Y\right] .
$$


Remark 2.6. The map $M_{F}^{\circ} G \rightarrow G$ associated to a local immersion of stacks is not generally separated, though this should cause the reader no concern, since intersection theory is valid even on non-separated schemes and stacks. In fact, even those operations of [7] which require a so-called finite parametrization may be carried out on arbitrary Deligne-Mumford stacks which are of finite type over a field (no such operations show up in this paper). This is so thanks to the proof, [5] (10.1), that every Deligne-Mumford stack of finite type over a field possesses a finite parametrization, i.e., admits a finite surjective map from a scheme.

Remark 2.7. The reader who wishes greater generality may see easily that all results in this section are valid in the setting of Artin stacks which are locally of finite type over an excellent Dedekind domain.

\section{Equivariance for tangent bundle action}

We continue to work with stacks which are locally of finite type over some base field. A special case of Proposition 2.3 is when $i: X \rightarrow Y$ is a local immersion of smooth Deligne-Mumford stacks. Suppose $j: Y^{\prime} \rightarrow Y$ is a local immersion, with $Y^{\prime}$ an arbitrary Deligne-Mumford stack. Recall that the local immersion $j$ gives rise to a natural group action of $j^{*} T_{Y}$ on $C_{Y^{\prime}} Y$. In short, the action is given locally (say $Y$ is an affine scheme and $Y^{\prime}$ is the closed subscheme given by the ideal $I$ ) by considering the action of $\left.T_{Y}\right|_{Y^{\prime}}$ on $\operatorname{Spec} \operatorname{Sym}\left(I / I^{2}\right)$ induced by the map $I / I^{2} \rightarrow \Omega_{Y}^{1}$ and proving ([2], Lemma $3.2)$ that the normal cone $\operatorname{Spec} \bigoplus I^{k} / I^{k+1}$ is invariant under the group action.

If we let $N_{X} Y$ be the normal bundle to $X$ in $Y$ and denote simply by $N$ its pullback to $X^{\prime}:=X \times_{Y} Y^{\prime}$, then $C_{X} Y \times_{Y} C_{Y^{\prime}} Y$ is identified with $N \times X_{X^{\prime}} i^{*} C_{Y^{\prime}} Y$. Viewing $T_{Y^{\prime}}$ as a subbundle of $j^{*} T_{Y}$, we have the natural action of $\left.T_{Y^{\prime}}\right|_{X^{\prime}}$ on $i^{*} C_{Y^{\prime}} Y$. This plus the trivial action on $N$ gives an action of $\left.T_{Y^{\prime}}\right|_{X^{\prime}}$ on $N \times_{X^{\prime}} i^{*} C_{Y^{\prime}} Y$.

Theorem 3.1. The rational equivalence between $\left[C_{i^{*} C_{Y^{\prime}} Y} C_{Y^{\prime}} Y\right]$ and $\left[N \times_{X^{\prime}}\right.$ $\left.C_{X^{\prime}} Y^{\prime}\right]$ produced in the proof of Proposition 2.3 is invariant under the action of $\left.T_{Y^{\prime}}\right|_{X^{\prime}}$ on $N \times{ }_{X^{\prime}} C_{X^{\prime}} Y^{\prime}$ described above.

As a consequence, the rational equivalence descends to a rational equiv- 
alence on the stack quotient $\left[N \times_{X^{\prime}} i^{*} C_{Y^{\prime}} Y /\left.T_{Y^{\prime}}\right|_{X^{\prime}}\right]$. This fact is exploited in [2] (Lemma 5.9, where the authors invoke the incorrect stronger claim appearing in Proposition 3.5 that the rational equivalence is equivariant for the bigger group $\left.T_{Y}\right|_{X^{\prime}}$.

Proof. The question is local, so we may assume $Y$ is an irreducible scheme, smooth and of finite type over the base field, $X$ is an smooth irreducible closed subscheme of $Y$, and $Y^{\prime}$ is a closed subscheme of $Y$. If $X \subset Y^{\prime}$ then the group action is trivial and there is nothing to prove, so we assume the contrary.

Lemma 3.2. Let $Y$ be a smooth irreducible scheme of finite type over a field $k$, of dimension $n$, let $X$ be a smooth irreducible closed subscheme of $Y$ of codimension $d$, and let $Y^{\prime}$ be a closed subscheme of $Y$ such that $X \not \subset Y^{\prime}$. Let $x$ be a closed point of $Y^{\prime} \cap X$. Then, after suitable base change by a finite separable extension of the base field, and after shrinking $Y$ to a neighborhood of $x$ in $Y$, there exists an étale map $f: Y \rightarrow \mathbb{A}^{n}$ such that $X$ maps into a linear subspace of $\mathbb{A}^{n}$ of codimensiond and such that $Y^{\prime} \rightarrow f\left(Y^{\prime}\right)$ is étale.

Proof. We may assume $x$ is a $k$-valued point, and moreover that $Y$ sits in $\mathbb{A}^{l}$ with $X=\mathbb{A}^{l-d} \cap Y$ (for suitable $l$ ). We may take $x$ to be the origin of $\mathbb{A}^{l}$. We consider as candidates for $f$ all linear functions mapping the flag $\mathbb{A}^{l-d} \subset \mathbb{A}^{l}$ into the flag $\mathbb{A}^{n-d} \subset \mathbb{A}^{n}$.

Those $f$ with $f_{*}: T_{x, Y} \rightarrow T_{f(x), \mathbb{A}^{n}}$ surjective form an open subscheme $U$ of $\mathbb{A}^{n l-d l+d^{2}}$. Define locally closed subschemes $V_{1}$ and $V_{2}$ of $Y \times U$ by

$$
V_{1}=\left\{(y, f) \in\left(Y^{\prime} \cap X \backslash\{x\}\right) \times U \mid f(y)=0\right\}
$$

and

$$
V_{2}=\left\{(y, f) \in\left(Y^{\prime} \backslash X\right) \times U \mid f(y)=0\right\},
$$

and let $\mathrm{pr}_{2}: Y \times U \rightarrow U$ be projection. A dimension count using the fact that $X \not \subset Y^{\prime}$ gives $\operatorname{dim}\left(V_{1}\right)<\operatorname{dim}(U)$ and $\operatorname{dim}\left(V_{2}\right)<\operatorname{dim}(U)$, and hence $\left.U \backslash \overline{\left(p r_{2}\left(V_{1}\right)\right.} \cup \overline{p r_{2}\left(V_{2}\right)}\right)$ is nonempty.

Since the rational equivalence of the proof of Proposition 2.3 commutes with étale base change, we are reduced by the Lemma to the case where $Y=\mathbb{A}^{n}$ and $X=\mathbb{A}^{m}$ (as a linear subspace of $\mathbb{A}^{n}$ ). Now we need the 
Key Observation. Assume $Y=\mathbb{A}^{n}$ and $Y^{\prime}$ is a closed subscheme of $Y$. Identify $T_{Y}$, as a group scheme over $Y$, with the additive group $\mathbb{A}^{n}$. Then there is a group action of $\mathbb{A}^{n}$ on $\widetilde{M}_{Y^{\prime}}^{\circ} Y$ (which we define to be the fiber of $M_{Y^{\prime}}^{\circ} Y \rightarrow \mathbb{P}^{1}$ over $\mathbb{A}^{1}$ ) which restricts to the natural action of $T_{Y}$ on $C_{Y^{\prime}} Y$.

Indeed, we let $\mathbb{A}^{n}$ act on $Y \times \mathbb{A}^{1}$ by

$$
\left(a_{1}, \ldots, a_{n}\right) \cdot\left(x_{1}, \ldots, x_{n}, t\right)=\left(x_{1}+t a_{1}, \ldots, x_{n}+t a_{n}\right) .
$$

By the universal property of blowing up, this extends uniquely to an action of $\mathbb{A}^{n}$ on $\widetilde{M}_{Y^{\prime}}^{\circ} Y$. If $Y^{\prime}$ is given by the ideal $\left(f_{1}, \ldots, f_{k}\right)$, and if we view $\widetilde{M}_{Y^{\prime}}^{\circ} Y$ as the closure of the graph of $\left(f_{1} / t, \ldots, f_{k} / t\right): Y \times\left(\mathbb{A}^{1} \backslash\{0\}\right) \rightarrow \mathbb{A}^{k}=$ Spec $k\left[z_{1}, \ldots, z_{k}\right]$, then the action is given coordinatewise by

$$
\mathbf{a}=\left(a_{1}, \ldots, a_{n}\right): z_{i} \mapsto z_{i}+\left(f_{i}(\mathbf{x}+t \mathbf{a})-f_{i}(\mathbf{x})\right) / t,
$$

so at $t=0$ we recover $z_{i} \mapsto z_{i}+D_{\mathbf{a}} f_{i}(\mathbf{x})$. This is the natural action of $T_{Y}$ on $C_{Y^{\prime}} Y$.

Concluding the proof of equivariance, we observe that $\widetilde{M}_{\mathbb{A}^{m}}^{\circ} \mathbb{A}^{n}$ fits into the fiber diagram

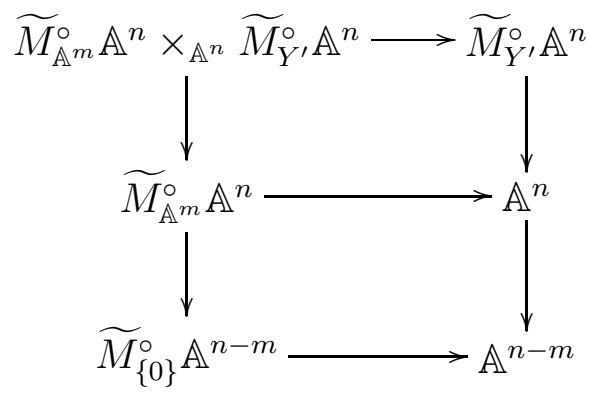

and now the action from the Key Observation of $\mathbb{A}^{m} \subset \mathbb{A}^{n}$ on $\widetilde{M}_{Y^{\prime}}^{\circ} Y$, plus the trivial action of $\mathbb{A}^{m}$ on $M_{\{0\}}^{\circ} \mathbb{A}^{n-m}$, combine to give a group action of $\mathbb{A}^{m}$ on $\widetilde{M}_{X}^{\circ} Y \times{ }_{Y} \widetilde{M}_{Y^{\prime}}^{\circ} Y$. The function $\widetilde{M}_{X}^{\circ} Y \times{ }_{Y} \widetilde{M}_{Y^{\prime}}^{\circ} Y \rightarrow \mathbb{A}^{1} \times \mathbb{A}^{1}$ which is used in Corollary 1.10 is invariant for this $\mathbb{A}^{m}$-action. Since the rational equivalence of the proof of Proposition 2.3 is compatible with smooth pullback, we get the desired equivariance result. 


\section{Appendix: unramified morphisms}

We give an elementary algebraic proof of the following fact.

Lemma 4.1. Let $S \rightarrow T$ be an unramified morphism of affine schemes which are of finite type over a base field $k$. Then there exists a commutative diagram of affine schemes

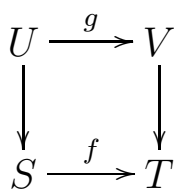

such that the vertical maps are étale surjective and such that $g$ is a closed immersion.

This fact plus the local nature of the property of being unramified gives us Lemma 2.2.

Proof. Say $S=\operatorname{Spec} A, T=\operatorname{Spec} B$, and $f$ is given algebraically by $f^{*}: B \rightarrow$ $A$. Recall that for $f$ to be unramified means that for every maximal ideal $\mathfrak{p}$ of $A$ with $\mathfrak{q}=f(\mathfrak{p})$, we have $f^{*}(\mathfrak{q}) \cdot A_{\mathfrak{p}}=\mathfrak{p} A_{\mathfrak{p}}$, and the induced field extension $B / \mathfrak{q} \rightarrow A / \mathfrak{p}$ is separable.

Case 1: The induced field extension $B / \mathfrak{q} \rightarrow A / \mathfrak{p}$ is an isomorphism. Then, if $x_{1}, \ldots, x_{n}$ are generators of $A$ as a $k$-algebra, we may write

$$
x_{i}=f^{*}\left(t_{i}\right)+w_{i}
$$

with $t_{i} \in B$ and $w_{i} \in \mathfrak{p}$, for each $i$. Since $f$ is unramified, we have

$$
w_{i}=\sum_{j=1}^{m_{i}} \frac{f^{*}\left(y_{i j}\right) p_{i j}}{q_{i}}
$$

for some $y_{i j} \in \mathfrak{q}, p_{i j} \in A$, and $q_{i} \in A \backslash \mathfrak{p}$.

Choose representative polynomials $P_{i j}$ and $Q_{i}$ in $k\left[X_{1}, \ldots, X_{n}\right]$ such that 
$P_{i j}\left(x_{1}, \ldots, x_{n}\right)=p_{i j}$ and $Q_{i}\left(x_{1}, \ldots, x_{n}\right)=q_{i}$. Let

$$
\begin{array}{r}
V=\operatorname{Spec} B\left[X_{1}, \ldots, X_{n}\right] /\left(X_{1} Q_{1}-t_{1} Q_{1}-\sum_{j=1}^{m_{1}} y_{1 j} P_{1 j}, \ldots,\right. \\
\left.X_{n} Q_{n}-t_{n} Q_{n}-\sum_{j=1}^{m_{n}} y_{n j} P_{n j}\right),
\end{array}
$$

and define $g: S \rightarrow V$ by $B \stackrel{f^{*}}{\rightarrow} A$ and $X_{i} \mapsto x_{i}$, and let $\varphi: V \rightarrow T$ be given by inclusion of $B$. Then $g$ is a closed immersion, and by the Jacobian criterion $\varphi$ is étale in some neighborhood of $g(\mathfrak{p})$.

Case 2: The field extension $B / \mathfrak{q} \rightarrow A / \mathfrak{p}$ is separable. Let $k^{\prime}$ be the maximal subfield of $A / \mathfrak{p}$ which is separable over $k$, and make the étale base change Spec $k^{\prime} \rightarrow$ Spec $k$ to get $f^{\prime}: S^{\prime} \rightarrow T^{\prime}$. Now $S^{\prime}$ has an $A / \mathfrak{p}$-valued point which maps to $\mathfrak{p} \in S$, and since $k^{\prime}$ together with $B / \mathfrak{q}$ generates all of $A / \mathfrak{p}$ we are now in the situation of Case 1 .

\section{References}

[1] M. Artin, Versal deformations and algebraic stacks, Invent. Math. 27 (1974), 165-189.

[2] K. Behrend and B. Fantechi, The intrinsic normal cone, Invent. Math. 128 (1997), 45-88.

[3] W. Fulton, Intersection Theory, Springer-Verlag, Berlin, 1984.

[4] H. Gillet, Intersection theory on algebraic stacks and $Q$-varieties, $J$. Pure Appl. Algebra 34 (1984), 193-240.

[5] G. Laumon and L. Moret-Bailly, Champs Algébriques, Prépubl. math. de l'Université de Paris-Sud, 1995.

[6] D. Quillen, Higher algebraic K-theory, I, in Algebraic K-theory (Seattle, Wash., 1972), pp. 85-147, Lect. Notes in Math. 341, Springer-Verlag, Berlin, 1973. 
[7] A. Vistoli, Intersection theory on algebraic stacks and on their moduli spaces, Invent. Math. 97 (1989), 613-670.

Department of Mathematics

University of Chicago

5734 S. University Avenue

Chicago, IL 60637

kresch@math.uchicago.edu 